\title{
LA BÚSQUEDA DE LA EXCELENCIA ACADÉMICA: DE LA CUDCA (1983) A LA U.D.C.A (2008)
}

\author{
Germán Anzola Montero ${ }^{1}$
}

Hacer referencia a lo ocurrido en la U.D.C.A durante sus 25 años de existencia como institución de educación superior privada, requiere la definición de períodos o etapas, que a mi juicio, podrían ser las siguientes:

- Creación y establecimiento de las bases para un proyecto educativo en la década de los 80 .

- De lo univocacional o uniprofesional a la universalización del conocimiento.

- Consolidación de procesos para la verdadera Universidad del siglo XXI.

\section{CREACIÓN Y ESTABLECIMIENTO DE LAS BASES PARA UN PROYECTO EDUCATIVO EN LA DÉCADA DE LOS 80}

Mediante la Ley $8^{\mathrm{a}}$ del 24 de enero de 1979, se otorgaron algunas facultades extraordinarias para establecer la naturaleza, las características y los componentes del Sistema de Educación Postsecundaria; con base en ella, el Presidente de la República de Colombia expidió el Decreto número 80 del 22 de enero de 1980. Este fue el fundamento jurídico que el grupo de fundadores, liderados por el Licenciado Alberto Gómez Moreno, tuvieron en cuenta para la creación de la Corporación Universitaria de Ciencias Agropecuarias CUDCA. La citada norma generó muchas inquietudes entre los universitarios del país, pues Colombia había carecido, hasta entonces, de principios rectores que le dieran un real y verdadero ordenamiento a la educación superior.

Para el proyecto de la CUDCA fueron concluyentes varios aspectos, entre los cuales resalto la clara y evidente definición de objetivos y la adecuada organización para el cumplimiento del proyecto educativo, sometido a

${ }^{1}$ Biólogo, M.Sc., Esp. Educación Superior. Rector Universidad de Ciencias Aplicadas y Ambientales U.D.C.A. consideración del gobierno nacional, representado en ese entonces por el ICFES. En este sentido, los fundadores decidieron crear una institución en la modalidad universitaria, cuya Misión se fundamentara en la formación ética, técnica y científica de profesionales, que respondieran a las necesidades presentes y futuras de nuestro país.

Determinante para este proyecto fue lo estipulado en el Decreto 80, en el sentido que se podrían organizar programa académicos, mediante un currículo integrado o por ciclos. Bajo este modelo y acogido por la CUDCA, se ofrecieron los programas de Medicina Veterinaria y de Zootecnia, a través de dos ciclos, de tal manera que, al término del primero, el educando estaría preparado para el ejercicio de una actividad tecnológica y conducente al título de Tecnólogo en Sanidad Animal o Tecnólogo en Producción Animal, para posteriormente ingresar a un segundo ciclo y así obtener el Título, a nivel universitario, en Medicina Veterinaria o Zootecnia, respectivamente. Para este período, se requería acreditar el título de Tecnólogo en la respectiva área (artículos 25, 2631 y 32 del Decreto 80 de 1980), demostrar haber laborado en el campo de su especialidad y cumplir con los demás requisitos que establecían los decretos reglamentarios. Es así que sus dos primeras promociones estuvieron sujetas a esta normativa.

Esta definición fundacional fue causa de serias incomprensiones por parte de estudiantes, de profesores y de directivas, generando, a mediados del año 1986, una grave crisis, que fue superada con la intervención del ICFES, situación que trajo, como secuela, el retiro del Rector, personal directivo, personal docente y un buen número de estudiantes. Como consecuencia fue necesario replantear el Proyecto Educativo Institucional, a partir de la consagración de un Plan de Desarrollo, que incorporara las verdaderas responsabilidades sociales y académicas y que asumiría una institución de educación superior privada, como prestadora de un Servicio Público Cultural, en lo que respecta a la DOCENCIA, la INVESTIGACIÓN y la EXTENSIÓN. 
El no haber tenido claridad sobre los aspectos y las funciones antes mencionados determinó la pérdida prácticamente de más dos años en sus ejecuciones reales. Hoy diría, por la complejidad de lo acontecido, que este aniversario corresponderá a los veintidós años y medio.

Como se puede observar fueron duras experiencias pero a su vez generaron procesos de aprendizaje. El primero de ellos y fundamental en las sociedades modernas es el relacionado con la Responsabilidad Social que debe asumir una institución de educación superior privada cuando le es otorgada la correspondiente Personería Jurídica, por parte del Ministerio de Educación Nacional. Y, por otro lado, la Obligación Social con el Estado Colombiano, con la comunidad académica y con las sociedades internacionales, la cual se debe expresar en la conducta, en los fundamentos y en los criterios para desarrollar su compromiso académico: una docencia responsable de alta calidad y pertinente; una investigación con alto rigor científico, en función de las necesidades y los requerimientos del país y acorde con la formación de sus estudiantes en las diferentes modalidades y una extensión articulada con los sectores sociales y productivos, para contribuir con el desarrollo científico y tecnológico, en consonancia con la Nación y con la sociedad colombiana.

Todo lo anterior procede bajo el marco de la Misión institucional y soportado en una nómina de directivos y de personal académico, significativamente cualificada. Así considero, transcurrió la primera década de vida institucional.

\section{DE LO UNIVOCACIONAL O UNIPROFESIONAL A LA UNIVERSALIZACIÓN DEL CONOCIMIENTO}

Con la llegada de los años noventas, determinante para el desarrollo y el futuro de la institución, puedo decir con mucho orgullo, que gracias a una tenaz perseverancia y una enorme voluntad, la situación de prosperidad intelectual y académica de nuestra incipiente Universidad, produjo hechos de significativa trascendencia, como los siguientes:

- A nivel nacional, la promulgación de la Ley 30 de 1992, la cual reformó las normas existentes y estableció un nuevo ordenamiento para la creación y el funcionamiento de instituciones de educación superior.
- A nivel institucional, la temprana y absurda desaparición de tres de sus fundadores, entre ellos su líder Alberto Gómez Moreno, a quien hoy recordamos y agradecemos la visión de futuro para crear la U.D.C.A.

- La determinación de lograr el reconocimiento institucional, como Universidad, implicó el trabajo en nuevas áreas del conocimiento, en función de la investigación, la innovación, el trabajo nter., multi y transdisciplinario, de la extensión y de la internacionalización. Dicho reconocimiento, se materializó el 29 de diciembre de 2004.

- Como consecuencia de los procesos de globalización, la internacionalización ha sido determinante para el trabajo conjunto de las comunidades académicas, frente a las nuevas realidades del contexto nacional e internacional y el establecimiento de esquemas innovadores para la gestión y el financiamiento de las Universidades.

- La necesidad de participar y trabajar en redes académicas y científicas, que favorezcan el establecimiento de alianzas y obtener mayor calidad en el desarrollo y en el progreso institucional, así como el uso imprescindible de nuevas tecnologías y sistemas de información y comunicaciones.

- La imperiosa necesidad de establecer sistemas de aseguramiento de la calidad para lograr la excelencia académica.

- La consecución de las mejores relaciones Universidad - Sociedad - Empresa.

- La profesionalización, como fundamento, para ejercer las responsabilidades académicas y administrativas en el ejercicio universitario.

- Los nuevos retos que determina la responsabilidad social de las instituciones de educación superior, para demostrar que la eficacia y la modernidad deben estar en armonía con una Universidad que trabaje por el desarrollo humano y sostenible de la sociedad del siglo XXI.

Los anteriores hechos dieron pie para proponer la permanente revisión de los principios, los propósitos y los programas que había establecido la U.D.C.A y promover el establecimiento de una Universidad moderna, acorde con los tiempos presentes y futuros. Con la actual estructura orgánica, las Vicerrectorías y las Decanaturas orientan y guían el destino de la institución, 
las cuales, a través de las diferentes facultades, ponen en ejecución los programas, a nivel de pregrado y de postgrado.

Como hecho destacable es preciso mencionar el fomento de la investigación científica de alto nivel y la definición de estrategias para impulsar las actividades de extensión, con miras a favorecer la educación permanente, las relaciones con la sociedad, con el gobierno y con los sectores productivos del país. De igual manera, no puede soslayarse la permanente interacción con los exalumnos y la preocupación para trabajar por la preservación de los procesos culturales, en el orden nacional e internacional.

\section{CONSOLIDACIÓN DE PROCESOS PARA LA VERDADERA UNIVERSIDAD DEL SIGLO XXI}

Con la experiencia acumulada, los últimos tres años de existencia de la U.D.C.A han marcado y determinado su modernización, mediante procesos de fortalecimiento y de consolidación, al aplicar mecanismos de planeación y de revisión de normas, de estatutos y de procedimientos, asociados a la redefinición de la Visión, la Misión y el Proyecto Educativo Institucional, lo cual ha redundado en reformas académicas, administrativas y financieras.

Las nuevas facultades de Química, Química Farmacéutica, Ciencias Ambientales y las de la Escuela de Ciencias Económicas Administrativas y Financieras, se podría señalar como el logro más representativo para consolidar el desarrollo académico, a nivel de pregrado. A ello se agrega y a corto plazo, la creación de las facultades en el área de Educación y de Artes.

Ahora, nuestros principales compromisos son la puesta en funcionamiento de los programas de Maestría en Ciencias Veterinarias, en Ciencias Agrícolas, en Ciencias Ambientales y en Agroforestería y el afianzamiento del Centro de Educación Virtual, que favorecerá, sin lugar a dudas, una mayor actividad en la modalidad de educación a distancia. Será prioridad de la Rectoría, el fortalecimiento de la Educación Continua, constituida como tal, desde hace más de diez años. Con el apoyo y compromiso de las facultades, ampliar su oferta educativa a nivel nacional $\mathrm{y}$, de ser posible, internacionalmente. De igual forma, los programas de pregrado y de postgrado serán ofrecidos en otras ciudades del país, dando prioridad a la implementación de la facultad de Ciencias Agropecuarias del Caribe, con sede en diferentes ciudades de la citada región.

Debo destacar la continuidad, a partir del presente año, del Programa de Desarrollo Profesoral que, conjuntamente con la actualización del Estatuto Docente, ha de mejorar, significativamente, las condiciones del personal docente de la U.D.C.A y, bajo este contexto, consolidar el concepto de comunidad académica, prevista en el Plan de Desarrollo 2006 - 2010.

Lograr el financiamiento del Proyecto de Desarrollo Arquitectónico permitirá el mejoramiento de la planta física, optimizando las condiciones para la labor docente, investigativa y de extensión.

Capítulo especial merece el impulso de la capacidad editorial en la U.D.C.A, representada en el incremento de la edición de libros y la cualificación de nuestra publicación seriada, representada en la revista Actualidad $\mathcal{E}$ Divulgación Científica. Otras formas de difusión del conocimiento generado por profesores y estudiantes son los referentes a los medios electrónicos que serán estimuladas e incrementadas en los próximos años.

Nuestro desarrollo institucional determina ajustes en los procesos de gestión y de financiamiento, los cuales se complementarán con las siguientes estrategias, que desde ya estamos ejecutando: la implementación de Sistemas de Gestión de Calidad en todos los procesos académicos y administrativos y la diversificación de fuentes de financiamiento, como complemento a los ingresos por concepto de matrículas.

A partir de los procesos de auto-evaluación y de acreditación tanto institucional como de programas y con la creación de la Unidad de Aseguramiento de la Calidad, identificaremos estrategias para cualificar nuestra Gestión Universitaria, en aspectos prioritarios, como la estructura orgánica, el Gobierno y Gobernabilidad institucional (liderazgo, toma decisiones, distribución de responsabilidades), la Misión, la Visión, el PEI, los Sistemas de Información, la promoción e identidad institucional, los sistemas de control, el financiamiento, la planeación universitaria y la administración de talento humano.

Especial importancia tendrán las actividades complementarias que generen fuentes de financiamiento 
para la U.D.C.A, en adición a los ingresos por matrícula. Entre las ideas que tenemos en mente, cabe mencionar: la creación de unidades de negocios, las donaciones, los servicios de consultoría y la asesoría y la investigación contratada, acciones que serán ejecutadas de inmediato. De igual forma, se buscará el uso eficiente de los activos y demás recursos institucionales y la eventual venta de aquellos que sean improductivos.

Como conclusión, quiero afirmar que el futuro de la U.D.C.A está en la COMPETITIVIDAD que pueda proyectar en el conjunto de las Universidades Colombianas. Esto significa que la U.D.C.A no deberá disminuir el número de aspirantes en sus procesos de ingreso, tanto a nivel de pregrado como de postgrado y deberá estar en capacidad de incrementar sus admisiones y aumentar los índices de retención de estudiantes, con el ofrecimiento de más y mejores servicios académicos, financieros y de bienestar social universitario. Igualmente, la competencia investigativa, de manera permanente, deberá mostrar crecimiento en los indicadores de gestión de dicha actividad (financiamiento de sus proyectos, publicaciones, patentes, grupos de investigación, obtención de reconocimientos, entre otros). Estos niveles de competitividad serán significativos en todo lo que determinen sus proyectos de proyección a la comunidad.

Para lograr esta COMPETITIVIDAD, la U.D.C.A debe generar las necesarias y propicias condiciones, como infraestructura, recursos económicos, humanos, físicos, seguridad y confianza, a sus actuales y potenciales usuarios. Ser competitivos significa identificar y aumentar nuestras mejores y mayores fortalezas, con el propósito de alcanzar la siempre anhelada EXCELENCIA ACADÉMICA.

Recibido: Marzo 31 de 2008

Aceptado: Abril 28 de 2008 